\title{
Original Research \\ Circulating microRNAs in the prediction of BAV aortopathy: do the expression patterns correlate between blood and aortic tissue?
}

\author{
Shiho Naito ${ }^{1, *}$, Tatiana Sequeira-Gross ${ }^{2}$, Johannes Petersen ${ }^{1,3}$, Inken Detlef ${ }^{1}$, Marco Sachse ${ }^{1}$, \\ Tanja Zeller ${ }^{3,4}$, Hermann Reichenspurner ${ }^{1,3}$, Evaldas Girdauskas ${ }^{2,3}$ \\ ${ }^{1}$ Department of Cardiovascular Surgery, University Heart and Vascular Center Hamburg, 20246 Hamburg, Germany \\ ${ }^{2}$ Department of Cardiothoracic Surgery, Augsburg University Hospital, 86150 Augsburg, Germany \\ ${ }^{3}$ German Center of Cardiovascular Research (DZHK), Partner Site Hamburg/Lübeck/Kiel, 22761 Hamburg, Germany \\ ${ }^{4}$ Department of Cardiology, University Center of Cardiovascular Science, University Heart and Vascular Center Hamburg, 20246 Hamburg, Germany \\ *Correspondence: s.naito@uke.de (Shiho Naito) \\ Academic Editor: Wayne L. Miller \\ Submitted: 23 November 2021 Revised: 9 January 2022 Accepted: 11 January 2022 Published: 8 February 2022
}

\begin{abstract}
Background: The limitation of aortic size-based criteria is gradually recognized in the prediction of aortic events especially in bicuspid aortic valve (BAV) cohorts, while most aortic events happen in patients with proximal aortic diameters $<50 \mathrm{~mm}$. Circulating microRNAs (miRs) have been addressed as a novel tool to improve risk stratification in patients with different aortopathies. We aimed to elucidate the correlation between peripheral whole blood and aortic tissue miRs in order to prove the potential availability as a biomarker in the clinical routine. Methods: All patients who received elective aortic valve repair/replacement \pm proximal aortic replacement to BAV disease (n $=65,2013-2018)$ were prospectively included. The expression of 10 miRs (miR-1, miR-17, miR-18a, miR-19a, miR-20a, miR-21, miR-106a, miR-133a, miR-143 and miR-145) was analyzed in the intraoperatively acquired aortic tissue as well as in the peripheral blood before the surgery. Results: We found a significant correlation between circulating miRs in the peripheral blood and aortic tissue levels of miR-21 ( $\mathrm{r}=0.293, p=0.02)$, miR-133a $(\mathrm{r}=0.43, p=0.02)$, miR-143 $(\mathrm{r}=0.68, p<0.001)$, and $\mathrm{miR}-145(\mathrm{r}=0.68, p<0.001)$. Further, the multivariate logistic regression analysis revealed an association between blood and aortic tissue miR-143 levels each other (Odds Ratio [OR] 1.29, 95\% Confidence Interval [CI] 1.11-1.67, $p=0.02$; OR 1.36, 95\% CI 1.19-2.01, $p=0.03$, respectively) and a blood/aortic miR-143 level to dilated aorta (OR 3.61, 95\% CI 1.62-9.02, $p=0.01$; OR 2.92, 95\% CI 1.81-7.05, $p=0.02$, respectively). Conclusions: Our study demonstrates a significant correlation between peripheral whole blood and aortic tissue miRs, confirming the hypothesis that circulating miRs may reflect remodeling processes in the proximal aorta in bicuspid aortopathy patients.
\end{abstract}

Keywords: bicuspid aortopathy; biomarker; microRNA

\section{Introduction}

Bicuspid aortic valve (BAV) associated aortic aneurysm, so-called "BAV-aortopathy" is accompanied with an increased risk of aortic events. Aortic dissection in BAV cohorts in small size were sporadically reported and the accuracy of size-based criteria for these patients are gradually questioned in the prediction of future aortic emergencies [1,2]. Individual decision making tools combinated with aortic size are still missing. Blood microRNAs (miRs) are potential biomarkers to improve risk stratification in BAV-aortopathy [3]. Circulating miRs have been demonstrated to reflect a wide spectrum of systemic diseases (e.g., malignancy, inflammation, cardiomyopathy) [4-9], and therefore the specificity of circulating miRs for prediction of aortic disease is still controversial [9]. In other words, there is still no evidence that down-or upregulated expression patterns of circulating miRs reflect specifically the pathophysiological changes in the aortic wall (i.e., vascular remodeling).

Our object was to analyze the correlation patterns of circulating miRs in patients with bicuspid aortic valve
(BAV) associated aortopathy and the miRs in the aortic tissue.

\section{Methods}

We reviewed our institutional prospective BAV registry to identify all patients who received aortic valve repair/replacement \pm proximal aortic replacement as elective procedure due to BAV disease with or without concomitant aortic aneurysm between 2013 and 2018. Bicuspid or unicuspid aortic valve was implicated preoperatively if only two normal commissures resulting in two aortic valve cusps or one cusp are detected in two-dimensional transthoracic echocardiographic short-axis, respectively. Further transesophageal echocardiography was also performed intraoperatively. The final verification of bicuspidity and the fusion type was descripted intraoperatively by the surgeon.

Proximal aortic dimensions were assessed by means of transthoracic/transesophageal echocardiography, computed tomography (CT) or magnetic resonance imaging (MRI).

Ascending aorta wall tissue specimens were obtained from the greater and lesser curvature in all patients at the time of surgery. Peripheral whole blood for miRs analysis 
was sampled just before the surgery (i.e., during anesthesia induction). The expression of $10 \mathrm{miRs}$ was analyzed in the intraoperatively obtained aortic tissue and peripheral whole blood (miR-1, miR-17, miR-18a, miR-19a, miR-20a, miR21, miR-106a, miR-133a, miR-143 and miR-145), all published previously in association with aortopathies $[3,10]$. Primary study endpoint was the correlation between the miRs in the aortic tissue and the peripheral whole blood.

Medical Ethical Committee approval for this prospective study was granted at the University Medical Center Hamburg Eppendorf and all patients gave their informed written consent.

\subsection{Tissue miRNA analysis}

Resected aortic tissue specimens were immediately frozen in liquid nitrogen and stored at $-80{ }^{\circ} \mathrm{C}$ and homogenized using miRNeasy Mini Kit Qiagen (Qiagen, Hilden, Germany). The RNA concentration was measured on the NanoDrop N1000 System (peqlab, Erlangen, Germany) and $10 \mathrm{mg}$ of RNA was used for miRs analysis. The RNA quality was measured using an Agilent 2100 Bioanalizer (Aglient Technology, Inc., Santa Clara, CA, USA). For normalization, miR-16 was used which is known to be elevated in malignant diseases [11]. In our study cohort, neither malignancy nor systemic inflammatory disease was included. cDNA synthesis and miR analysis were performed using the TaqMan Advanced miRNA cDNA Synthesis Kit (Thermo Fisher Scientific, Waltham, MA, USA) on Quant Studio 7 Flex real-time PCR detection system (Thermo Fisher Scientific, Waltham, MA, USA). The Ct values were normalized to miR-16 by the formula $2^{--(C t[m i R N A]--C t[m i R-16])}$ for $\mathrm{Ct}<40$. In case for $\mathrm{Ct}$ values $\geq 40$, miR was considered as undetermined.

\subsection{Peripheral whole blood microRNA}

Blood samples for miRs analysis were collected prior to surgery. Whole blood was collected in PaxGene Blood RNA tubes and were frozen at $-20{ }^{\circ} \mathrm{C}$ over night, and subsequently at $-80{ }^{\circ} \mathrm{C}$ for long-term storage until RNA isolation. Total RNA was isolated using the PAXgene Blood miRNA kit (Qiagen, Hilden, Germany) on a QIAcube system (Qiagen) according to the manufacturer's recommendations. Concentration of isolated RNA was measured on the NanoDrop N1000 System (peqlab), and $10 \mathrm{ng}$ of RNA was used for miR analysis. The RNA quality was measured using an Agilent 2100 bioanylizer (Agilent Technology, Inc., Santa Clara, CA, USA). For further analysis, only samples with RNA integrity number above seven were included. The same $10 \mathrm{miRs}$ in tissue miRs analyses were selected. cDNA synthesis and miR analysis were performed using the TaqMan Advanced miRNA cDNA synthesis kit and commercially available TagMan assay specific for each miRNA (Thermo Fischer Scientific) on a 7900 HT real-time system. In the case $\mathrm{Ct}$ values were $\geq 40$, miRs were considered as undetermined.

\subsection{Statistics}

Continuous variables are reported as mean \pm standard deviation. Categorical variables are presented as percentages. All statistical analyses were performed using the IBM SPSS 24.0 software (IBM Corp, New York City, NY, USA). All $p$-values $<0.05$ were considered statistically significant. Normal distribution of miRs values was confirmed by SPSS Shapiro Wilk test. Two-sided $t$-test was used for comparisons of parametric variables. Furthermore, $\chi^{2}$-test and Fisher's exact (as appropriate) test were used for categorical variables. Using the Pearson correlation coefficient, Correlation between miR values in the aortic tissue and the blood was analyzed in the whole study cohort. Further, multivariate logistic regression analysis was performed to find associations between blood and aortic tissue levels of miR-143 and clinical variables including age, gender, aortic diameter $(\geq 40 \mathrm{~mm})$, and predominant aortic stenosis. These variables of clinical interest were chosen if it achieved a $p$-value $<0.10$ at previous univariate analysis.

We performed post-hoc study power calculation based on the correlation analysis of peripheral whole blood vs. aortic tissue miR-133a expression. Study power was $88.2 \%$ based on the sample size of 49 patients and the probability of $\alpha$ error of $5 \%$.

\section{Results}

Our study cohorts included a total of 47 consecutive BAV and 2 unicuspid aortic valve (UAV) patients referred to our institution for elective aortic valve surgery with or without concomitant proximal aortic surgery. Table 1 summarizes the baseline characteristics of the study population. The mean aortic diameter was $37 \pm 8 \mathrm{~mm}$ and one third of our study population (37\%) had a maximal proximal aortic diameter $\geq 40 \mathrm{~mm}$. The proximal aortic procedure was required in $8(16 \%)$ patients.

Our biomarker analysis included 10 specific miRs, all previously reported in association with aortopathies (Table 2). The strongest correlation was found for miR-21 ( $\mathrm{r}$ $=0.293, p=0.02)$, miR-133a $(\mathrm{r}=0.43, p=0.02)$ (Fig. 1$)$, miR-143 ( $\mathrm{r}=0.68, p<0.001$ ) (Fig. 2), and miR-145 (r $=$ $0.68, p<0.001)$.

We included multivariate logistic regression analysis to find significant associations between blood and aortic tissue levels of miR-143 (Supplementary Tables 1,2). These results showed a significant correlation between the blood to aortic miR-143 level (Odds Ratio [OR] 1.36, 95\% Confidence Interval [CI] 1.19-2.01, $p=0.03)$ and aortic tissue to blood miR-143 level (OR 1.29, 95\% CI 1.11-1.67, $p=$ $0.02)$ each other. Further, dilated aortic diameter $(\geq 40 \mathrm{~mm})$ was significant correlated to blood miR-143 level (OR 3.61, 95\% CI 1.62-9.02, $p=0.01)$ and to aortic miR-143 level (OR 2.92, 95\% CI 1.81-7.05, $p=0.02$ ), respectively. 
Table 1. Baseline variables.

\begin{tabular}{lc}
\hline Variables & $\mathrm{n}=49$ \\
\hline Age, years, mean $\pm \mathrm{SD}$ & $57 \pm 10$ \\
Male, $\mathrm{n}(\%)$ & $33(67)$ \\
Hypertension, $\mathrm{n}(\%)$ & $28(57)$ \\
Diabetes mellitus, $\mathrm{n}(\%)$ & $3(6)$ \\
Hyperlipoproteinemie, $\mathrm{n}(\%)$ & $25(51)$ \\
Aortic diameter, mm, mean $\pm \mathrm{SD}$ & $37 \pm 8$ \\
Aortic diameter $\geq 40$ mm, $\mathrm{n}(\%)$ & $18(37)$ \\
LVEF, \%, mean \pm SD & $57 \pm 8$ \\
Predominant aortic stenosis, $\mathrm{n}(\%)$ & $43(88)$ \\
Predominant aortic insufficiency, $\mathrm{n}(\%)$ & $14(29)$ \\
Bicuspid aortic valve, $\mathrm{n}(\%)$ & $47(96)$ \\
$\quad \mathrm{R}-\mathrm{L}$ fusion, $\mathrm{n}(\%)$ & $10(20)$ \\
$\quad \mathrm{R}-\mathrm{N}$ fusion, $\mathrm{n}(\%)$ & $12(24)$ \\
$\quad \mathrm{L}-\mathrm{N}$ fusion, $\mathrm{n}(\%)$ & $3(6)$ \\
Unicuspid aortic valve, $\mathrm{n}(\%)$ & $2(4)$ \\
aortic valve replacement, $\mathrm{n}(\%)$ & $47(96)$ \\
aortic valve repair, $\mathrm{n}(\%)$ & $2(4)$ \\
concomitant proximal aortic surgery, $\mathrm{n}(\%)$ & $8(16)$ \\
\hline
\end{tabular}

LVEF, left ventricular ejection fraction; R-L, right and left coronary cusp; R-N, right coronary and non-coronary cusp; $\mathrm{L}-\mathrm{N}$, left coronary and non-coronary cusp.

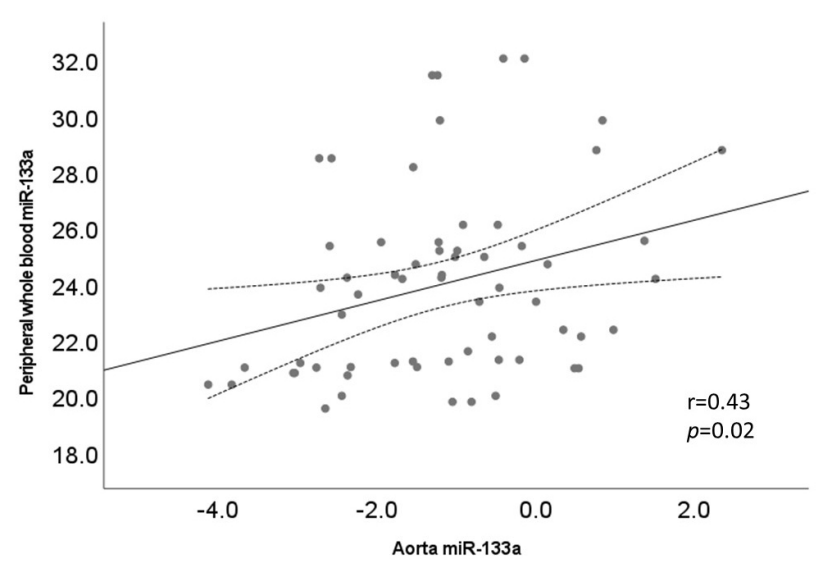

Fig. 1. Correlation between blood and aorta miR-133a. A significant correlation between a peripheral whole blood miR-133a and aorta miR-133a was detected.

In our study cohort, neither malignancy nor systemic inflammatory disease was included. Three patients for each two specimens (= six aortic tissue specimens) had reduces systolic left ventricular ejection function under $45 \%$.

\section{Discussion}

Our study demonstrates a significant linear correlation between peripheral whole blood and aortic tissue levels of miR-21, miR-133a, miR-143 and miR-145. Further, a significant correlation between blood/aortic miR-143 lev-
Table 2. Correlation analyses between aortic tissue and peripheral whole blood miRs.

\begin{tabular}{lll}
\hline miRNAs & correlation & $p$-value \\
\hline miR-17 & $\mathrm{r}=-0.012$ & $p=0.924$ \\
miR-18a & $\mathrm{r}=-0.173$ & $p=0.182$ \\
miR-19a & $\mathrm{r}=-0.091$ & $p=0.499$ \\
miR-20a & $\mathrm{r}=-0.174$ & $p=0.172$ \\
miR-21 & $\mathbf{r}=\mathbf{0 . 2 9 3}$ & $\boldsymbol{p}=\mathbf{0 . 0 2 0}$ \\
miR-133a & $\mathbf{r}=\mathbf{0 . 4 3 0}$ & $\boldsymbol{p}=\mathbf{0 . 0 2 0}$ \\
$\operatorname{miR}-143$ & $\mathbf{r}=\mathbf{0 . 0 6 8}$ & $\boldsymbol{p}<\mathbf{0 . 0 0 1}$ \\
$\operatorname{miR}-145$ & $\mathbf{r}=\mathbf{0 . 6 8 0}$ & $\boldsymbol{p}<\mathbf{0 . 0 0 1}$ \\
\hline
\end{tabular}

MiR-1 and miR-106a were undetermined. Bold values are relevant in results. MiR, microRNA.

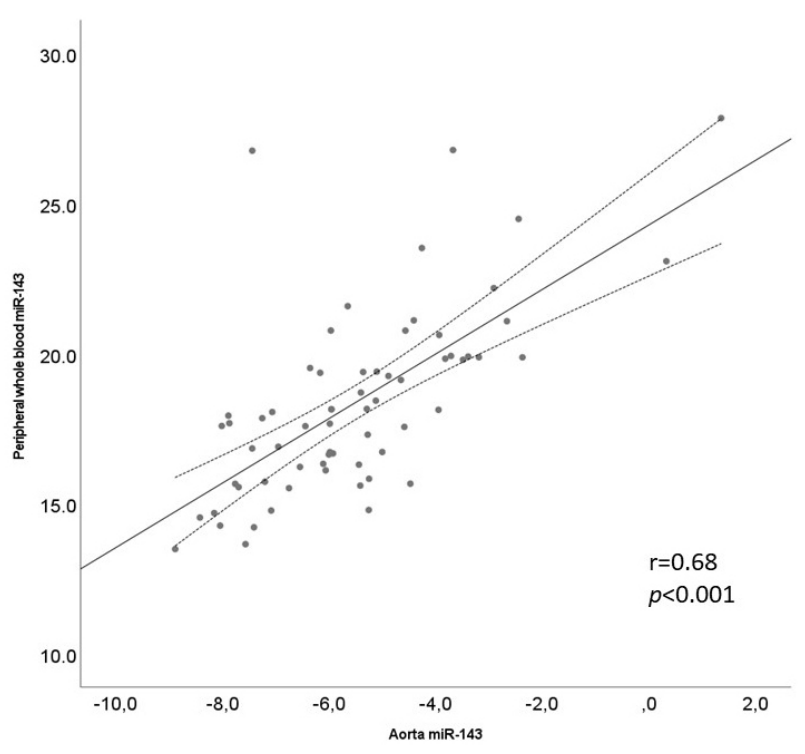

Fig. 2. Correlation between blood aorta miR-143. A significant correlation between a peripheral whole blood miR-143 and aorta miR-143 was detected.

els and dilated aorta was also confirmed. Therefore, these expression patterns of peripheral whole blood miRs can be used to reflect the remodeling processes in the proximal aorta in aortopathy patients (i.e., vascular remodeling) and these peripheral whole blood miRs have a potential to become individualized biomarkers for aortopathy prediction.

In former series, we showed the correlation between aortic miRs and matrix metalloproteinases (MMP)/tissue inhibitors of matrix metalloproteinases (TIMP) [12] and between peripheral whole blood miRs and aortic diameter [10]. Wu et al. [13] performed a paired comparison of the miR expression between severely dilated vs. normal appearing, less-dilated aortic samples. The authors found an association between differential regulation of the miR-17 gene cluster and the predisposition to dilatation through the dysregulation of TIMP-MMP pathway [13]. Further, Lu et 
al. [14] reported miR-34a and miR-125 down-regulation of MMP-2 expression on bicuspid aortic wall specimen, which is related to wall shear stress. Pasta et al. [15] succeeded to show the association of the strain at the mid ascending aorta with upregulation of plasma levels of miR-320 and miR-26a and the wall shear stress with a downregulation of miR-29a. Maleki et al. [16] proposed a new mechanism of vascular remodeling through endothelial/epithelial mesenchymal transition in bicuspid aortopathy. They demonstrated a miR-200c/ZEB1/ZEB2 negative feedback loop in BAV aorta prior to aneurysm.

The current standard management of aneurysm is based on maximal aortic diameter detected by routine imaging modalities. However, there is nowadays some concern and evidence suggesting that relying on a single aortic diameter measurement may be too restrictive and not so reliable [2]. Further, especially in bicuspid aortopathy, conventional aortic size-based decision criteria are of very limited value to provide accurate information regarding the risk evaluation given the heterogeneity of the BAV cohorts [1]. Therefore, novel diagnostic tools, which are independent of size-based criteria, are urgently needed to predict the aortopathy progression and to define the appropriate time point for surgical intervention.

Thanks to extensive research efforts and enthusiasms in the area of BAV aortopathy, a lot of pathophysiological aspects have been revealed in the last decade. However, a reliable prediction of the aortopathy progression is still very premature. Several pathophysiogical pathways and the associated proteins/genes have been detected, which can be potentially used as diagnostic or predictive tools in the bicuspid aortopathy in the next future [17].

MiRs are endogenously expressed single-stranded non-coding RNA molecule containing 18-22 nucleotides. MiRs regulate the degradation of translational suppression of specific target messenger RNAs, and and as a result maintain various biological processes. Their expression patterns can be significantly affected in many human diseases. MiRs can be exported or released by cells into the blood stream in highly stable forms [18]. Due to their reproducibility and easy accessibility, miRs have been introduced as potential biomarkers in many human diseases.

Previous reports revealed the up- or downregulation of several miRs including miR-15, -21, -24, -29, -30, -133, $-143,-145,-155,-193,-205,-223,-933$ on aortic aneurysm [19]. To our best knowledge, there is still no aortopathyspecific miR, which no other diseases or health circumstances effects on the measurement.

Compared to extracellular matrix proteins, such as MMP or TIMP, and mRNA, miRs are more stable and degrade much slower, making them detectable and measurable in serum and even in formalin-fixed and paraffinembedded tissues [20]. Ikonomidis et al. [3] mentioned in their study that there was no specific correlation between plasma and tissue MMPs and TIMPs levels. Then, the half- life of the analytes is variable, the differences in plasma and tissue storage and handling affect the ability to accurately measure the analyte [3]. Therefore, the wide availability and stability of miRs make them attractive as potential biomarkers as compared to other biomolecules.

MiR-133a encoding gene is located on chromosome 18. The previously published data show the downregulation of expression in some malignancies, like gastric cancer [4], esophageal squamous cell carcinoma [5], and pancreatic cancer [6]. The clinical hypothesis suggests that the down-regulation of miR-133a expression may enhance the progression of malignant disease, tumor recurrence, and aggressive metastases, thus leading to poor clinical outcome [21]. Further, myocardial miR-133a is downregulated in dilated cardiomyopathy [7] and shows increased expression in left ventricular reverse remodeling [8]. The increased expression level of blood miR-133a after acute myocardial infarction is also reported and the clinical use as new diagnostic marker is expected [22].

The miR-143 encoding genes are located on human chromosome 5 [23]. MiR-143 is known as an important tumor suppressor in variety of neoplasms including solid tumors and B-cell malignancies [24]. Nowadays, miR-143 attracts increasingly the attention as an effectual approach and a potential new therapeutic target to treat neoplasms [24]. In the cardiovascular field, miR-143/-145 cluster is predominantly expressed in vascular smooth muscle cells and play an essential role in various diseases, including essential hypertension [25], atherosclerosis [26], pulmonary arterial hypertension [27], and coronary artery disease [28]. Several results from animal experiments support the therapeutical potential of targeting miR-143/-145 in these cardiovascular diseases. Human clinical trials are now under consideration [29].

MiR-21 regulates cell proliferation, differentiation, and apoptosis [30], which affect tumor biological behaviors, including growth, invasion and migration. Former reports presented higher expression in pulmonary carcinoma [30], acute myeloid leukemia [31] and so on. MiR-21 is up-regulated in cardiomyocytes during the fibrotic process [32]. Therefore, in aortic valve stenosis, both myocardial and blood levels of miR-21 are higher than control, which correlates with aortic pressure gradient and fibrosis [32].

Interestingly, Ikonomidis et al. [3] demonstrated differential plasma expression profiles of MMPs, TIMPs and miRNAs in BAV patients vs. patients with tricuspid aortic valve. In bicuspid aortopathy, receiver operator characteristic curve analysis showed highest accuracy in the combination pattern of miR-143, miR-133a, miR-145, MMP2 and TIMP-2. These findings support our current results in that blood miR-133a, miR-143 and miR-145 correlate significantly with the corresponding miRs in the aortic tissue. However, they failed to demonstrate the correlation between plasma and aortic tissue miRNAs. This discrepancy between our results and those from the abovemen- 
tioned study could be the different storage and processing protocols. The authors mentioned the need for refinement of the protocols in their report [3].

\section{Potential limitations of the circulating miRs}

Even though circulating miRs have a great potential to become biomarkers for aortopathy, there are still some important concerns. The specificity of circulating miRs for detection of aortopathy progression remains still questionable. On the other hand, miRs expression patterns in the aortic tissue samples provide a great deal of information on the pathophysiological mechanisms of the underlying diseases. However, this expression signature in the tissue does not necessarily correlate with the expression patterns in the bloodstream [3]. Further, unlike tissue-specific miRs, the expression patterns of circulating miRs might be influenced by several other tissues and organs whereby limiting their specificity for one particular disease [9]. The expression profile of circulating miRs may, therefore, be a result of pathophysiological processes in the multiple tissues, in case of complex diseases. In this regard, our current study provides important evidence of the positive correlation between circulating and aortic tissue miRs.

From the future perspective, longitudinal evaluation of changes in the miRs expression patterns and the correlation with the aortopathy progression in the same patient would be of a major interest. Such data would contribute to the validation of miRs as reliable biomarkers in the aortopathy progression.

Several limitations of our study should be noted. First, the size of our study cohort is quite limited. The study included no control group. Further, our study cohort is young and male dominant, which is a typical finding for the bicuspid group. We could not evaluate the impact of malignancy or other systemic diseases. Therefore, the further evaluation of other potential factors that may influence miRs expression is warranted in large prospective study.

Finally, due to the heterogeneity of bicuspid aortopathy, other potential pathways of the aortopathy progression and identification of other potential biomarkers should be clarified.

\section{Conclusions}

Our study indicates that peripheral whole blood miRs expression correlates significantly with aortic tissue miRs expression. This finding confirms our clinical hypothesis that circulating miRs may reflect vascular remodeling processes in the bicuspid aortopathy patients.

\section{Author contributions}

Conceptualization-EG, TZ and SN; methodologyTS-G and JP; software-TZ; validation-TZ; formal analysis-ID and MS; investigation-ID and MS;
resources-TZ; data curation-JP; writing- original draft preparation- $\mathrm{SN}$; supervision-HR; project administration-HR. All authors contributed to editorial changes in the manuscript. All authors read and approved the final manuscript.

\section{Ethics approval and consent to participate}

The study was conducted in accordance with the Declaration of Helsinki, and the protocol was approved by the ethics committee of the Medical Associaton Thuringia, Nr. 23333/2014/146. Each individual patient gave a written informed consent.

\section{Acknowledgment}

We would like to express our gratitude to all those who helped us during the writing of this manuscript. Thanks to all the peer reviewers for their opinions and suggestions.

\section{Funding}

This research received no external funding.

\section{Conflict of interest}

The authors declare no conflict of interest.

\section{Supplementary material}

Supplementary material associated with this article can be found, in the online version, at https://www.imrpre ss.com/journal/RCM/23/2/10.31083/j.rcm2302047.

\section{References}

[1] Girdauskas E, Borger MA. Bicuspid Aortic Valve and Associated Aortopathy: an Update. Seminars in Thoracic and Cardiovascular Surgery. 2013; 25: 310-316.

[2] Girardi LN, Lau C, Gambardella I. Aortic dimensions as predictors of adverse events. The Journal of Thoracic and Cardiovascular Surgery. 2021; 161: 1193-1197.

[3] Ikonomidis JS, Ivey CR, Wheeler JB, Akerman AW, Rice A, Patel RK, et al. Plasma biomarkers for distinguishing etiologic subtypes of thoracic aortic aneurysm disease. The Journal of Thoracic and Cardiovascular Surgery. 2013; 145: 1326-1333.

[4] Li C, Li X, Gao S, Li C, Ma L. MicroRNA-133a Inhibits Proliferation of Gastric Cancer Cells by Downregulating ERBB2 Expression. Oncology Research Featuring Preclinical and Clinical Cancer Therapeutics. 2017; 25: 1169-1176.

[5] Kano M, Seki N, Kikkawa N, Fujimura L, Hoshino I, Akutsu Y, et al. MiR-145, miR-133a and miR-133b: Tumor-suppressive miRNAs target FSCN1 in esophageal squamous cell carcinoma. International Journal of Cancer. 2010; 127: 2804-2814.

[6] Wang Z. Diagnostic performance for declined microRNA-133a in pancreatic cancer. Journal of Cellular Biochemistry. 2020; 121: 3882-3886.

[7] Wang Y, Li M, Xu L, Liu J, Wang D, Li Q, et al. Expression of Bcl-2 and microRNAs in cardiac tissues of patients with dilated cardiomyopathy. Molecular Medicine Reports. 2017; 15: 359 365.

[8] Dziewięcka E, Totoń-Żurańska J, Wołkow P, Kołton-Wróż M, Pitera E, Wiśniowska-Śmiałek S, et al. Relations between circulating and myocardial fibrosis-linked microRNAs with left 
ventricular reverse remodeling in dilated cardiomyopathy. Advances in Clinical and Experimental Medicine. 2020; 29: 285293.

[9] Haider BA, Baras AS, McCall MN, Hertel JA, Cornish TC, Halushka MK. A critical evaluation of microRNA biomarkers in non-neoplastic disease. PLoS ONE. 2014; 9: e89565.

[10] Girdauskas E, Neumann N, Petersen J, Sequeira-Gross T, Naito $\mathrm{S}$, von Stumm $\mathrm{M}$, et al. Expression Patterns of Circulating MicroRNAs in the Risk Stratification of Bicuspid Aortopathy. Journal of Clinical Medicine. 2020; 9: E276.

[11] Cho WCS. MicroRNAs as therapeutic targets for lung cancer. Expert Opinion on Therapeutic Targets. 2010; 14: 1005-1008.

[12] Naito S, Petersen J, Sequeira-Gross T, Neumann N, Duque Escobar J, Zeller T, et al. Bicuspid aortopathy - molecular involvement of microRNAs and MMP-TIMP. Biomarkers. 2020; 25: 711-718.

[13] Wu J, Song H, Li S, Guo J, Tsang K, Tumiati L, et al. Progressive Aortic Dilation is Regulated by miR-17-Associated miRNAs. Journal of the American College of Cardiology. 2016; 67: 2965-2977.

[14] Lu Y, Zhang L, Tao H, Sun X, Zhao Y, Xia L, et al. Two MicroRNAs, miR-34a and miR-125a, Are Implicated in Bicuspid Aortopathy by Modulating Metalloproteinase 2. Biochemical Genetics. 2021. (in press)

[15] Pasta S, Agnese V, Gallo A, Cosentino F, Di Giuseppe M, Gentile G, et al. Shear Stress and Aortic Strain Associations with Biomarkers of Ascending Thoracic Aortic Aneurysm. The Annals of Thoracic Surgery. 2020; 110: 1595-1604.

[16] Maleki S, Cottrill KA, Poujade F-, Bhattachariya A, Bergman $\mathrm{O}$, Gådin JR, et al. The mir-200 family regulates key pathogenic events in ascending aortas of individuals with bicuspid aortic valves. Journal of Internal Medicine. 2019; 285: 102-114.

[17] Naito S, Hillebrand M, Bernhardt AMJ, Jagodzinski A, Conradi $\mathrm{L}$, Detter C, et al. The Value of Circulating Biomarkers in Bicuspid Aortic Valve-Associated Aortopathy. The Thoracic and Cardiovascular Surgeon. 2016; 66: 278-286.

[18] Tsui NBY, Ng EKO, Lo YMD. Stability of endogenous and added RNA in blood specimens, serum, and plasma. Clinical Chemistry. 2002; 48: 1647-1653.

[19] Moushi A, Michailidou K, Soteriou M, Cariolou M, Bashiardes E. MicroRNAs as possible biomarkers for screening of aortic aneurysms: a systematic review and validation study. Biomarkers. 2018; 23: 253-264.

[20] Chen X, Ba Y, Ma L, Cai X, Yin Y, Wang K, et al. Characterization of microRNAs in serum: a novel class of biomarkers for diagnosis of cancer and other diseases. Cell Research. 2008; 18: 997-1006.

[21] Nohata N, Hanazawa T, Enokida H, Seki N. MicroRNA-1/133a and microRNA-206/133b clusters: dysregulation and functional roles in human cancers. Oncotarget. 2012; 3: 9-21.

[22] Zhu L, Liu F, Xie H, Feng J. Diagnostic performance of microRNA-133a in acute myocardial infarction: a metaanalysis. Cardiology Journal. 2018; 25: 260-267.

[23] Weber C, Schober A, Zernecke A. MicroRNAs in arterial remodelling, inflammation and atherosclerosis. Current Drug Targets. 2010; 11: 950-956.

[24] Karimi L, Mansoori B, Shanebandi D, Mohammadi A, Aghapour M, Baradaran B. Function of microRNA-143 in different signal pathways in cancer: New insights into cancer therapy. Biomedicine \& Pharmacotherapy. 2017; 91: 121-131.

[25] Kontaraki JE, Marketou ME, Zacharis EA, Parthenakis FI, Vardas PE. Differential expression of vascular smooth musclemodulating microRNAs in human peripheral blood mononuclear cells: novel targets in essential hypertension. Journal of Human Hypertension. 2014; 28: 510-516.

[26] Wei Y, Nazari-Jahantigh M, Neth P, Weber C, Schober A. MicroRNA-126, -145, and -155: a therapeutic triad in atherosclerosis? Arteriosclerosis, Thrombosis, and Vascular Biology. 2013; 33: 449-454.

[27] Caruso P, Dempsie Y, Stevens HC, McDonald RA, Long L, Lu $\mathrm{R}$, et al. A role for miR-145 in pulmonary arterial hypertension: evidence from mouse models and patient samples. Circulation Research. 2012; 111: 290-300.

[28] Fichtlscherer S, De Rosa S, Fox H, Schwietz T, Fischer A, Liebetrau $\mathrm{C}$, et al. Circulating MicroRNAs in Patients with Coronary Artery Disease. Circulation Research. 2010; 107: 677-684.

[29] Zhao W, Zhao S, Zhao Y. MicroRNA-143/-145 in Cardiovascular Diseases. BioMed Research International. 2015; 2015: 531740.

[30] Sun M, Song J, Zhou Z, Zhu R, Jin H, Ji Y, et al. Comparison of Serum MicroRNA21 and Tumor Markers in Diagnosis of Early Non-Small Cell Lung Cancer. Disease Markers. 2016; 2016: 17.

[31] Moussa Agha D, Rouas R, Najar M, Bouhtit F, Fayyad-Kazan $\mathrm{H}$, Lagneaux L, et al. Impact of Bone Marrow miR-21 Expression on Acute Myeloid Leukemia T Lymphocyte Fragility and Dysfunction. Cells. 2020; 9: 1-18.

[32] Fabiani I, Pugliese NR, Calogero E, Conte L, Mazzanti MC, Scatena C, et al. MicroRNAs distribution in different phenotypes of Aortic Stenosis. Scientific Reports. 2018; 8: 9953. 